To investigate current practice in patient preparation prior to gastroscopy across the UK.

Methods A list of all Hospitals in the UK was obtained from the JAG website (391). Hospitals that did not perform endoscopy were excluded (14), as were Children's Hospitals (5) and private hospitals (165). The number of hospitals included was 207. A structured telephone survey was conducted with each endoscopy unit. The method of preparation prior to gastroscopy was established.

Results 193 (93\%) endoscopy units responded to the survey. 11 (5\%) endoscopy units declined to participate in the survey and $3(2 \%)$ of endoscopy units did not respond. Preparation prior to gastroscopy (\%) included:

1. $6 \mathrm{~h}$ nil by mouth (NBM) to food or clear fluids (38\%)

2. $6 \mathrm{~h} \mathrm{NBM}$ to food and NBM to clear fluids for $2 \mathrm{~h}(26 \%)$

3. NBM to food and clear fluids from midnight for morning lists with 6 h NBM for afternoon lists (13\%)

4. Nine different methods of preparation accounted for the remaining $23 \%$ of hospitals.

No hospital used a mucolytic drink for routine gastroscopy cases. Conclusion Current gastroscopy preparation regimes vary across the UK. Future studies should evaluate which preparation regime provides the best possible visualisation of the upper gastrointestinal tract.

Competing interests None declared.

\section{PTU-212 TO ANALYSE THE RISK OF STENT MIGRATION WITH THE NITI-S COVERED FLARED BILIARY STENT}

doi:10.1136/gutjnl-2012-302514c.212

'J K Moore, ${ }^{1} \mathrm{M}$ Davies, ${ }^{2} \mathrm{M}$ Sheridan. ${ }^{1}$ Hepatology, St James University Hospital, Leeds, UK; ${ }^{2}$ Radiology, St James University Hospital, Leeds, UK

Introduction Stent migration occurs in $6 \%-12 \%$ of patients undergoing biliary stenting ${ }^{1}$ The Niti-S fully covered metal stent (Taewoong Medical, Seoul) has a flared end which acts as antimigration. ${ }^{2}$ Few studies have evaluated the efficacy of these stents and are contradictory ${ }^{3}$ The aim of our study is to compare their migration rates, investigate contributory factors and patient outcomes.

Methods This was a retrospective cohort study. 32 Niti-S flared stents were placed between January 2010 and July 2011. Each of the patients' records were analysed. The indication for stent, size and whether there had been any previous endoscopic therapy was recorded. The cholangiograms were then re-reviewed by an experienced radiologist to assess stricture length and position of the stent proximal to the stricture.

Results 10 out of 32 stents (31\%) had migrated. Nine were placed for benign strictures and one for a malignant stricture. There was no significant difference between the stents that did and did not migrate comparing the length of stricture (mean $11.8 \mathrm{~mm}$ vs $12.4 \mathrm{~mm}$ ), or where the stent was placed (mean proportion of stent above the proximal end of the stricture $16 \mathrm{~mm}$ vs $17.5 \mathrm{~mm}$ ). There also appeared to be no association with previous endoscopic therapy: in 6 (60\%) of the procedures where the stents had migrated there had been previous therapy, compared to 14 of the remaining 22 procedures (64\%). 4 of the $9(44 \%)$ patients with migrated stents were subsequently admitted to hospital with cholangitis, compared to 1 patient who did not have a migrated stent.

Conclusion The flared end covered metal stents significantly migrate despite the theories behind the design. Direct cost implications of this should be sought. There appears to be no association between aetiology, length of stricture, previous endoscopic therapy or where the stent was placed and subsequent migration.

\section{Competing interests None declared.}

\section{REFERENCES}

1. Kullman E, Frozanpor F, Soderland C, et al. Covered versus uncovered selfexpandable nitinol stents in the palliative treatment of malignant distal biliary obstruction: results from a randomised, multicenter study. Gastrointest Endosc 2010; 72:915-23.

2. Park do H, Song TJ, Eum J, et al. EUS - guided hepaticogastrostomy with a fully covered metal stent as the biliary diversion technique for an occluded biliary stent after a failed ERCP (with videos). Gastrointest Endosc 2010;71:413-19.

3. Park do H, Lee SS, Lee $\mathrm{TH}$, et al. Anchoring flap versus flared end, fully covered selfexpandable metal stents to prevent migration in patients with benign biliary strictures: a multicentre, prospective, comparative pilot study (with videos). Gastrointest Endosc 2011; 73:64-70

4. Han YM, Gin GY, Lee SO, et al. Flared polyurethane covered self-expandable Nitinol stent for malignant biliary obstruction. J Vasc Interv Radiol 2003:14:1291-301.

\section{PTU-213 SELF EXPANDABLE METAL STENTS (SEMS) FOR OBSTRUCTING COLORECTAL CANCER IN ENGLAND: LINKAGE ANALYSIS OF HOSPITAL EPISODE STATISTICS}

doi:10.1136/gutjnl-2012-302514c.213

1J Geraghty, ${ }^{*} \mathrm{~S}$ Sarkar, ${ }^{2} \mathrm{M}$ Shawihdi, ${ }^{2} \mathrm{E}$ Thompson, ${ }^{2} \mathrm{M}$ Pearson, ${ }^{2,3} \mathrm{~K}$ Bodger. ${ }^{1}$ Gastroenterology, Royal Liverpool University Hospital, Liverpool, UK; ${ }^{2}$ Aintree Health Outcomes Partnership, University of Liverpool, Liverpool, UK; ${ }^{3}$ Gastroenterology, University Hospital Aintree, Liverpool, UK

Introduction Colorectal cancer (CRC) is the 4th commonest cancer worldwide. Hospital admission with large bowel obstruction occurs in $15 \%$ and requires urgent decompression. SEMS can provide palliative treatment in advanced disease (avoiding surgical defunctioning) or preoperative bridging to elective surgery for operable disease. We aimed to describe a national profile for incidence (activity) of SEMS, volumes per Trust, length of stay and rates of readmission, reintervention and mortality for CRC in England.

Methods We developed techniques within the SPSS software package to identify a 1-year cohort of incident cases of CRC, starting with a merged file of raw HES data for all care episodes in English hospitals for $2006 / 7$ and $2007 / 8$. We selected only patients with first coding of CRC in the middle 12 months (October-September), then extracted all their admissions within 6 months (before and after) of first cancer coding, ordering them chronologically and then screening to identify admissions for SEMS and surgical procedures. Linkage to death registry provided date of death. Patients with SEMS and no subsequent surgical resection were flagged as palliative patients and those with a subsequent resection as bridge patients.

Results Overall: 517 patients were identified nationally as having SEMS placement for obstructing CRC (mean age: 72.6 yrs [SD: 12.0]; 62.5\% male), with mean LOS of 7.9 [SD 11.3] days and overall mortality at $30 \mathrm{~d}(10.3 \%)$ and $90 \mathrm{~d}(18.0 \%)$. The $30 \mathrm{~d}$ emergency readmission rate was $15.1 \%$. SEMS were code by $122(81.3 \%)$ of acute Trusts in England, with volumes ranging from 1 to 24 per institution. Palliative group: $(n=421,81.4 \%$ of cases), mean LOS for index admission 9.2 [SD: 14.6] days and mortality at $30 \mathrm{~d}$ $(12.1 \%)$ and $90 \mathrm{~d}(21.2 \%)$. Emergency readmission within $30 \mathrm{~d}$ (17.8\%). Subsequent surgical colostomy coded in 9.5\%. Palliative procedures were recorded in 122 Trusts (Volumes: $1-13$ per institution), Bridge group: ( $n=96,18.6 \%$ of cases), mean LOS for index admission 9.5 [SD: 10.4] days and mortality at $30 \mathrm{~d}(2.1 \%)$ and $90 \mathrm{~d}$ (4.2\%). Emergency $30 \mathrm{~d}$ readmission (8.7\%). Colostomy coded as part of surgery in $33.4 \%$. Bridge procedures were coded in $48(32 \%)$ acute Trusts (Volumes: 1-12)

Conclusion Analysis of HES data suggests SEMS insertion in English hospitals is predominantly for palliative purposes and most cases selected for this intervention survive beyond 30 days and avoid operative decompression. The use of SEMS as a bridge to surgery 
was relatively uncommon and one third required a stoma at surgery. Variation between Trusts in coding quality is inevitable but the data suggest 1 in 5 institutions may lack provision for SEMS.

Competing interests J Geraghty: Grant/Research Support from: Cook Medical, S Sarkar: None declared, M Shawihdi: None declared, E Thompson: None declared, M Pearson: None declared, K Bodger: None declared.

\section{PTU-214 AN IN-VITRO STUDY TO ASSESS, AND IMPROVE, THE ACCURACY OF COLONIC POLYP SIZING AMONG NURSE ENDOSCOPISTS, TRAINEES AND CONSULTANT GASTROENTEROLOGISTS}

doi:10.1136/gutjnl-2012-302514c.214

J Geraghty, ${ }^{*}$ Z Raisi-Estabragh, M Gillon, P O’Toole, S Sarkar. Gastroenterology, Royal Liverpool University Hospital, Liverpool, UK

Introduction Knowing if polyps are larger than $10 \mathrm{~mm}$ is critical when determining colonoscopic surveillance strategies. Judging polyp size from the endoscopic view alone becomes important if polyps are not retrieved intact. Strategies based on deliberately discarding small polyps rely on accurate discrimination of polyp size but little is known about endoscopists ability to make this judgement. Our aim is to assess the accuracy of polyp size estimation using a novel in vitro model, comparing different professional groups and use of accessories to improve estimates.

Methods Nine endoscopists ( 3 consultants, 3 trainees and 3 nurse endoscopists) judged the size of 15 "polyps" made from modelling clay (size range $6-36 \mathrm{~mm}$ ) placed inside a colonoscopy training model (Koken Co Ltd, Tokyo). Polyps of different sizes were presented in random order. Size estimates were made using endoscopic visual assessment alone or by comparing the polyp to biopsy forceps or a $10 \mathrm{~mm}$ snare. A degree of confidence for each guess was recorded.

Results Consultants and trainees were significantly better than nurse endoscopists at judging whether the model polyps were larger or smaller than $10 \mathrm{~mm}(91.8 \%$ vs $79.2 \% \mathrm{p}<0.05)$. Overall, visual assessment alone had an accuracy of $78.8 \%$. Inaccuracy was largely due to underestimation of size. Use of accessories improved discrimination around the $10 \mathrm{~mm}$ threshold $(\mathrm{p}<0.05)$. The snare produced slightly better accuracy (87.9\%) than forceps $(83.8 \%)$ (NS). All professional groups expressed similar degrees of confidence in their estimates.

Conclusion In this model, medical endoscopists were better than nurse endoscopists in assessing the size of polyps. This may be because nurses in our study do not routinely perform polypectomy whereas doctors have all had the opportunity to learn from comparing the size of resected polyps with their original endoscopic assessment. Use of biopsy forceps or a snare improved size estimation and these may be helpful tools when teaching this important aspect of polyp assessment in vivo.

Competing interests J Geraghty Grant/Research Support from: Cook Medical, Z Raisi-Estabragh: None declared, M Gillon: None declared, P O'Toole: None declared, S Sarkar: None declared.

\section{PTU-215 ASSESSING COMFORT SCORES FOR CO2 INSUFFLATION AS COMPARED TO AIR IN FLEXIBLE SIGMOIDOSCOPY-A PROSPECTIVE AUDIT}

doi:10.1136/gutjnl-2012-302514c.215

J Tharakan, ${ }^{*}$ D Beesley, A Kelling, R Radzioch, I Tiwari. Endoscopy unit, Braintree Community hospital, Essex, UK

Introduction Patients experience of discomfort with Air insufflation during flexible sigmoidoscopy (FS) limits compliance and thus success of the procedure. There has been only one study ${ }^{1}$ which has shown that CO2 insufflation reduces discomfort as compared to Air in FS. Recently, we have been using CO2 insufflation for routine FS. We therefore conducted a prospective audit comparing the two modalities and to assess whether the use of $\mathrm{CO} 2$ during FS reduces discomfort both during and after the procedure using a standardised scoring system.

Methods 200 consecutive patients undergoing FS, commonly for rectal bleeding, altered bowel habit and abdominal pain were selected to either Air or CO2 insufflation. There were 100 patients (42 males) in the CO2 group and 100 patients (51 males) in the Air group. The ages ranged from 19 to 92 years in both the groups. Any history of previous abdominal surgery was also noted. Patients were asked to grade discomfort during the procedure, post procedure in the recovery room and on discharge. We used the standardised comfort score of Wong and Baker $(0==$ no discomfort and $10=$ extreme discomfort). Abdominal bloating was also assessed verbally after the procedure. Statistical analysis was done using Prism software.

Results The mean comfort scores for $\mathrm{CO} 2$ compared to Air during the procedure was 1.02 vs 1.93 ( $p=0.0006)$, postprocedure 0.54 vs $1.12(p=0.002)$ and on discharge 0.32 vs $0.8(p=0.0008)$ respectively. Abdominal bloating appeared to be less with $\mathrm{CO} 2$ as compared to Air on verbal questioning. No differences in comfort scores were observed with a history of previous abdominal surgery.

Conclusion This study has shown that $\mathrm{CO} 2$ insufflation reduces discomfort as compared to Air during FS, both during and after the procedure. Abdominal bloating was also significantly reduced. The use of CO2 will contribute to better public acceptance for FS, in particular for FS screening in colorectal cancer.

Competing interests None declared.

\section{REFERENCE}

1. Bretthauer M, Hoff G, Thiis-Evensen, et al. CO2 insufflation reduces discomfort due to flexible sigmoidoscopy. Scan J Gastroenterol 2002;37:1103-7.

\section{PTU-216 A SURVEY OF PATIENTS ATTITUDES TO COLONOSCOPY DEMONSTRATES HIGH VALUE FOR ENDOSCOPIST INTERACTION BUT NOT THE SINGLE SEX ENVIRONMENT}

doi:10.1136/gutjnl-2012-302514c.216

J McEntire, ${ }^{*}$ J Sahota, T Hyde, T Trebble. Portsmouth Hospitals NHS, Portsmouth, UK

Introduction Understanding patient attitudes to their medical experience is essential for optimising care and use of resources. This includes their interaction with their health practitioner and their healthcare environment. This study was undertaken to determine patient's preferences and expectations for outpatient colonoscopy, a common gastrointestinal procedure for which there is limited such data from the UK.

Methods Unselected patients attending for elective colonoscopy at a large District General Hospital on randomly selected days in October and November 2011 were invited to participate. Patients independently completed a composite, validated dedicated endoscopy questionnaire, with Likert scale anxiety-related and single sex environment questions and a 15-point preference (ranking) scale of aspects of endoscopy care that were considered most important (1) to least important (15) as contributing to a satisfactory experience. Qualitative and pilot studies were performed initially to confirm validity and reliability in the local population.

Results 217 out of 225 patients agreed to participate (96.4\%); male (49\%) and female (51\%), with mean age of 58 years (range 16-87 years). Mild to moderate anxiety was recorded in over $70 \%$ of 